Features of liver tissue remodeling in intestinal failure during and after weaning off parenteral nutrition

Kurvinen, Annika

2016-09

Kurvinen , A , Lohi , J , Sorsa , T , Jalanko , H \& Pakarinen , M P 2016 , ' Features of liver tissue remodeling in intestinal failure during and after weaning off parenteral nutrition ' , Surgery, vol. 160 , no. 3 , pp. 632-642 . https://doi.org/10.1016/j.surg.2016.04.025

http://hdl.handle.net/10138/224130

https://doi.org/10.1016/j.surg.2016.04.025

publishedVersion

Downloaded from Helda, University of Helsinki institutional repository.

This is an electronic reprint of the original article.

This reprint may differ from the original in pagination and typographic detail.

Please cite the original version. 


\title{
Features of liver tissue remodeling in intestinal failure during and after weaning off parenteral nutrition
}

\author{
Annika Mutanen, MD, PhD, ${ }^{\text {a Jouko Lohi, MD, PhD, }}{ }^{\mathbf{b}}$ Timo Sorsa, MD, PhD, ${ }^{\mathrm{c}}$ \\ Hannu Jalanko, MD, PhD, ${ }^{\mathbf{d}}$ and Mikko P. Pakarinen, MD, PhD, ${ }^{\mathbf{a}}$ Helsinki, Finland
}

Background. Intestinal failure is associated frequently with liver injury, which persists after weaning off parenteral nutrition. We compared features of liver remodeling in intestinal failure during and after weaning off parenteral nutrition.

Methods. Liver biopsies and serum samples were obtained from 25 intestinal failure patients at a median age of 9.7 years (interquartile range: 4.6-18) and from age-matched control patients. Seven patients had been receiving parenteral nutrition for 53 months (22-160), and 18 patients had been weaned off parenteral nutrition 6.3 years (2.4-17) earlier, after having received parenteral nutrition for 10 months (3.3-34). Expression of alpha-smooth muscle actin, collagen 1, proinflammatory cytokines, growth factors, and matrix metalloproteinases (MMPs) was measured.

Results. Significant increases in immunohistochemical expression of alpha-smooth muscle actin and collagen 1 were observed predominantly in portal areas and were similar to increases seen in patients currently receiving parenteral nutrition and in patients weaned off parenteral nutrition. Gene and protein expressions of alpha-smooth muscle actin and collagen were interrelated. Gene expression of ACTA2, encoding alpha-smooth muscle actin, was increased only in patients who were receiving parenteral nutrition currently. Comparable upregulation of interleukin-1 ( $\alpha$ and $\beta$ ), epidermal growth factor, integrin- $\beta 6$, and MMP9 gene expression was observed in both patient groups, irrespective of whether they were receiving parenteral nutrition currently. Liver expression and serum levels of TIMP1 and MMP7 were increased only in the patients on parenteral nutrition currently but were not increased after weaning off parenteral nutrition.

Conclusion. Intestinal failure is characterized by abnormal activation of hepatic myofibroblast and accumulation of collagen both during and after weaning off parenteral nutrition. Persistent transcriptional upregulation of proinflammatory and fibrogenic cytokines after weaning off parenteral nutrition suggests that factors other than parenteral nutrition may contribute to intestinal failureassociated liver disease. (Surgery 2016;160:632-42.)

From the Section of Pediatric Surgery, Pediatric Liver and Gut Research Group, Children's Hospital, ${ }^{a}$ the Department of Pathology, HUSLAB, ${ }^{b}$ the Department of Oral and Maxillofacial Diseases, ${ }^{c}$ and the Department of Pediatric Nephrology and Transplantation, Children's Hospital, ${ }^{d}$ Helsinki University Central Hospital, University of Helsinki, Helsinki, Finland

Supported by grants from the Finnish Pediatric Research Foundation, the Sigrid Juselius Foundation, and the Helsinki University Central Hospital research funds.

Accepted for publication April 18, 2016.

Reprint requests: Mikko P. Pakarinen, MD, PhD, Section of Pediatric Surgery, Pediatric Liver and Gut Research Group, Children's Hospital, Helsinki University Central Hospital, Stenbackinkatu 11, PO Box 281, 00029 HUS, Helsinki, Finland. E-mail: mikko.pakarinen@hus.fi.

0039-6060/\$ - see front matter

(c) 2016 Elsevier Inc. All rights reserved.

http://dx.doi.org/10.1016/j.surg.2016.04.025
INTESTINAL FAILURE-ASSOCIATED LIVER DISEASE (IFALD) is a major complication of prolonged parenteral nutrition $(\mathrm{PN})$ in children and adults and is the leading cause for intestinal transplantation. ${ }^{1-3}$ Accumulating experimental and clinical evidence suggest that disrupted intestinal function may play a central role in development and maintenance of IFALD in addition to the hepatotoxic effects of PN. ${ }^{4-7}$

Histologically, IFALD progresses from initial cholestasis and inflammation to fibrosis and steatosis, which persist years after cessation of $\mathrm{PN} .{ }^{8}$ Whether the persistent histologic liver injury after 
weaning off $\mathrm{PN}$ represents an inactive scar or an active, potentially destructive process remains unknown. This question has become highly relevant as increasing numbers of children with IF are successfully weaned off PN using modern multidisciplinary approaches without organ transplantation. 9,1

In mice, the combination of $\mathrm{PN}$ and lipopolysaccharide translocation induced by intestinal injury synergistically activates Kupffer cell expression of proinflammatory cytokines and fibrogenic growth factors. ${ }^{4,5}$ In accordance with experimental studies, PN-dependent children with IF display an overabundance of lipopolysaccharideproducing Proteobacteria in their intestinal microbiota in association with histologic liver injury, intestinal inflammation, and increased levels of serum proinflammatory cytokines. ${ }^{11}$ Liver fibrosis is observed with increasing frequency after extensive distal small intestinal resection in children ${ }^{6,8}$ and even without a history of PN administration in pigs, ${ }^{12}$ supporting an essential role of disturbed intestinal homeostasis in the pathogenesis of IFALD.

With activation by chronic injury, hepatic stellate cells (or myofibroblasts from other sources) transform into myofibroblast-expressing $\alpha$-smooth muscle actin (alpha-SMA), which secretes extracellular matrix components, mainly collagen $1 .{ }^{13}$ Progression of liver fibrosis occurs when liver fibrogenesis prevails over fibrolysis by matrix metalloproteinases (MMPs), resulting in excess extracellular matrix deposition accompanied with upregulation of MMP tissue inhibitors (TIMPs). Tissue remodeling in liver injury is mediated by a multitude of proinflammatory cytokines, growth factors, and adhesion molecules, whose expression has not been addressed previously in patients with IF. ${ }^{13}$

This study aimed to investigate comparatively hepatic tissue remodeling in pediatric-onset IF by evaluating expression of smooth muscle, collagen, proinflammatory cytokines, profibrotic growth factors, and MMPs. We hypothesized that IF is characterized by increased smooth muscle expression, accumulation of extracellular matrix, and upregulation of cytokines, fibrogenic growth factors, and MMPs both during PN delivery and after weaning off PN, possibly due to compromised intestinal function, which provides continuos injurious stimulus to the liver after cessation of PN.

\section{PATIENTS AND METHODS}

Ethics. This study was approved by the Helsinki University Central Hospital ethics committee (2/ $13 / 03 / 03 / 2010)$ and the Institutional Review
Board (57/2010, 12/2013). Written informed consent was received from all patients and control patients or their caregivers before any procedures were undertaken.

Patients and study design. All patients with pediatric-onset IF treated and followed up with our intestinal rehabilitation program after massive small intestinal resection or prolonged $\mathrm{PN}$ at Children's Hospital, Helsinki University Central Hospital, from 1984 to 2010 were reviewed. Of the 52 survivors $(93 \%), 38$ underwent ultrasoundguided liver biopsy and serum sampling during general anesthesia for gastroscopy as described previously $^{8} ; 25$ with sufficient liver biopsy material were included in this study.

Control patients. Control serum samples were obtained from healthy, age-matched (9.7 years [5.2-16], $\quad P=.693)$, day-operative patients $(n=74)$ without metabolic, gastrointestinal, or hepatobiliary disease. Age-matched (15.0 years [8.1-17], $P=.141)$ transplant donor livers $(n=22)$ were used as controls for liver immunohistochemistry. Control liver tissue for measurements of RNA expression was obtained from age-matched (7.2 years [4.5-13], $P=.585)$ patients $(n=8)$ who had undergone fine-needle liver biopsy as a part of a research study protocol (434/13/03/03/2008) during an operation for complicated cholelithiasis.

Baseline data. Baseline patient data, including composition of PN during the 3 months preceding liver biopsy, number of blood culture-positive septic episodes from birth to study date, and operative procedures, were collected from patient records (Table I). The percentage of predicted, age-adjusted small bowel length was calculated based on age-specific normal values. ${ }^{14}$ Blood samples were drawn after an overnight fast and were analyzed for platelets, plasma aminotransferases, glutamyl transferase, prealbumin, bilirubin, conjugated bilirubin, and tromboplastin time by routine hospital laboratory methods. The aspartate aminotransferase to platelet ratio index was calculated. ${ }^{15}$

Serum MMPs, TIMP1, and interleukin-1ß. Serum levels of MMP7, MMP8, MMP9, and their tissue inhibitor, TIMP1, and interleukin-1 $\beta$ (IL-1 $\beta$ ) were estimated in duplicate by commercially available, enzyme-linked immune sorbent assay (ELISA) kits as described previously. ${ }^{16}$ The kit for MMP7 (Quantikine; R\&D Systems, Inc, Minneapolis, MN), kits for MMP9 and TIMP1 (Biotrak; Amersham Biosciences UK Ltd, Buckinghamshire, UK), and the ELISA kit for IL-1 $\beta$ (Quantikine HS ELISA; R\&D Systems, Inc) were used according to the manufacturer's protocol. 
Table I. Patient characteristics

\begin{tabular}{|c|c|c|c|}
\hline Variable & All patients & Patients on PN currently & Patients weaned off $P N$ \\
\hline Patients $(n)$ & 25 & 7 & 18 \\
\hline Age (y) & $9.7(4.6-18)$ & $4.9(3.5-13)$ & $11(5.0-20)$ \\
\hline Gestational age (wk) & $36(31-40)$ & $38(35-40)$ & $35(27-40)$ \\
\hline Birth weight (g) & $3,220(1,260-3,560)$ & $3,260(2,530-4,040)$ & $2,980(850-3,550)$ \\
\hline Weight Z-score & $-0.9(-1.9$ to 0.0$)$ & $-0.5(-1.2$ to 0.8$)$ & $-0.9(-2.0$ to -0.5$)$ \\
\hline Height Z-score & $-0.5(-1.8$ to 0.2$)$ & $-0.3(-2.4$ to 0.1$)$ & $-0.8(-1.7$ to 0.4$)$ \\
\hline $\mathrm{BMI}^{*}$ & $18.6(15.9-20.2)$ & $18.9(18.6-21.4)$ & $17.2(15.1-19.9)$ \\
\hline Age at PN start (d) & $0(0)$ & $0(0)$ & $0(0)$ \\
\hline Duration of PN (mo) & $14(4.8-56)$ & $53(22-160) \dagger$ & $10(3.3-34)$ \\
\hline Short bowel syndrome $(n / \%)$ & $16 / 64$ & $3 / 43$ & $13 / 72$ \\
\hline \multicolumn{4}{|l|}{ Small bowel } \\
\hline $\mathrm{Cm}$ & $31(15-48)$ & $14(9-15) \dagger$ & $35(28-49)$ \\
\hline$\%$ & $25(11-34)$ & $11(6-12) \dagger$ & $25(17-39)$ \\
\hline Ileocecal valve $(n)$ & 8 & 2 & 6 \\
\hline Colon $(\%)$ & $80(64-100)$ & $100(72-100)$ & $80(61-95)$ \\
\hline Motility disorders $(n / \%)$ & $9 / 36$ & $4 / 57$ & $5 / 28$ \\
\hline \multicolumn{4}{|l|}{ Small bowel } \\
\hline $\mathrm{Cm}$ & $230(130-300)$ & $210(95-330)$ & $240(130-340)$ \\
\hline$\%$ & $100(48-100)$ & $71(45-97)$ & $100(68-100)$ \\
\hline Ileocecal valve $(n)$ & 4 & $0 \dagger$ & 4 \\
\hline Colon (\%) & $0(0-100)$ & $0(0) \dagger$ & $100(0.1-100)$ \\
\hline
\end{tabular}

*BMI-for-age (ISO-BMI) for children aged 2-18 years; BMI $\left(\mathrm{kg} / \mathrm{m}^{2}\right)$ for patients aged $>18$ years.

$\dagger$ Mann-Whitney $U$ test or Fisher exact test for comparison between patients on PN and patients weaned off PN, $P<.05$.

Data are frequencies or medians (interquartile range).

$\%$, Percentage of age-adjusted small bowel length; $P N$, parenteral nutrition; $B M I$, body mass index.

The MMP8 levels were estimated by a timeresolved immunofluorometric assay. The monoclonal MMP8-specific antibodies 8708 and 8706 (Medix Biochemica, Kauniainen, Finland) were used as a catching antibody and a tracer antibody, respectively. ${ }^{17}$ The coefficients of variation (\%) for MMP7, MMP8, MMP9, TIMP1, and IL-1 $\beta$ were 4.4 , $7.3,8.8,13.1$, and 7.3, respectively, and the detection limits were $0.016 \mathrm{ng} / \mathrm{mL}, 0.08 \mathrm{ng} / \mathrm{mL}$, $0.6 \mathrm{ng} / \mathrm{mL}, 1.25 \mathrm{ng} / \mathrm{mL}$, and $0.057 \mathrm{pg} / \mathrm{mL}$, respectively.

Liver biopsies and histology. Liver biopsies were fixed in formalin, embedded in paraffin, sliced, and stained with conventional stains. The liver biopsies were analyzed for fibrosis, cholestasis, portal inflammation, and steatosis by 2 experienced pediatric liver pathologists and the primary researcher, blinded to clinical data, until a consensus was reached. In addition to lobular $(0=$ absent, $1=$ present , and $2=$ prominent $)$ and overall fibrosis (Metavir fibrosis stage), cholestasis, portal inflammation, and steatosis were graded as described previously. ${ }^{8}$

Immunohistochemistry. Immunostainings were performed using freshly cut, $4-\mu \mathrm{m}$ sections of formalin-fixed and paraffin-embedded liver samples. The immunohistochemistry for collagen 1 and alpha-SMA was analyzed by the primary researcher blinded to clinical data. Monoclonal antibodies used for immunostaining were clone I8H5 (Abnova Corporation, Taipei City, Taiwan) for collagen 1, clone 1A4 (Dako, Glostrup, Denmark) for alpha-SMA, clone 151-7B2 (Merck Millipore; Merck KGaA, Darmstadt, Germany) for MMP7, and clone 63515 (R\&D Systems) for TIMP-1. We used a Leica DM RXA microscope (Leica microsystems GmbH, Wetzlar, Germany) for imaging of the immunostained sections, and an area that included an average of 8 representative portal areas was selected for imaging.

For collagen 1 and alpha-SMA, the proportion of antibody-positive area (eg, area fraction) was measured with image analysis Software (Image J; Rasband WS, US National Institutes of Health, Bethesda, MD; [http://imagej.nih.gov/ij/]). MMP7 staining of bile ducts $(0=$ no staining, $1=$ weak staining, $2=$ moderate staining, $3=$ strong staining) and TIMP-1 staining of the periportal ( $0=$ no staining, $1=$ stained $)$ and perisinusoidal hepatocytes $(0=$ no staining, $1=<25 \%$ of hepatocytes, $2=25-50 \%$ of hepatocytes, $3=>50 \%$ of hepatocytes) were graded visually. All histologic and immunohistologic analyses were performed without knowledge of the clinical history of the subjects. 
RNA isolation and analysis of gene expression. Liver tissue specimens were embedded in RNA later-solution (Ambion; Life Technologies, Thermo Fisher Scientific Inc, Waltham, MA) and frozen until analyzed. RNA was extracted using a kit (RNeasy Mini Kit; Qiagen, Frederick, MD). RNA integrity was assessed spectrophotometrically. Gene expression was analyzed in triplicate by quantitative, real-time polymerase chain reaction (PCR), using a PCR array (Human Fibrosis RT $^{2}$ Profiler PCR Array; Qiagen SABiosciences) on a sequence detection system (ABI 7700; PerkinElmer Life Sciences, Boston, MA) according to the manufacturer's instructions. The primer for MMP7 was a product from Qiagen. Quantification of target gene mRNA expression was performed using the $\Delta \Delta \mathrm{Ct}$ method and was expressed after normalization to housekeeping genes and relative to controls.

Statistical analysis. Data are median and interquartile range or mean \pm standard error of the mean unless stated otherwise. For multiple comparisons, the Kruskal-Wallis test was used followed by a post hoc Mann-Whitney $U$ test when the Kruskal-Wallis test reached statistical significance. For pairwise comparisons, the Mann-Whitney $U$ test or Fisher exact test was performed. Associations were tested with Spearman rank correlation.

\section{RESULTS}

Patient characteristics. The baseline patient characteristics are shown in Table I. Etiology of IF included short bowel syndrome (necrotizing enterocolitis, $n=6$, small bowel atresia, $n=6$, midgut volvulus, $n=4)$, and intestinal motility disorders (chronic intestinal pseudo-obstruction, $n=5$, extensive aganglionosis of Hirschsprung's disease, $n=4)$. Seven patients had been receiving PN for a median of 53 months (22-160). They received $50 \%$ (17-95, range: $7-100)$ of the total energy supply parenterally in a median of $6(5-7) \mathrm{PN}$ infusions per week. Of the parenteral, nonprotein energy supply, $77 \%$ (64-83) or $4.4(3.0-8.1) \mathrm{g} / \mathrm{kg} / \mathrm{d}$ was given as glucose and $13 \%(8-29)$ as fat in 6 patients. The 6 patients received $0.3(0.2-0.9)$ $\mathrm{g} / \mathrm{kg} / \mathrm{d}$ PN fat from olive oil-based lipid emulsion (Clinoleic; Baxter, Helsinki, Finland). Eighteen patients had weaned off $\mathrm{PN}$ a median of 6.3 years (2.5-17) earlier, after receiving PN for 10 months (3.3-34).

Liver histology and biochemistry. Overall, 20 of 25 patients $(80 \%)$ had abnormal liver histology, with fibrosis in $17(68 \%)$, steatosis in $13(52 \%)$, portal inflammation in $2(8 \%)$, and cholestasis in 1 (4\%) (Table II). The frequency and grade of histologic liver fibrosis and steatosis were not significantly different between patients on PN and patients weaned off PN (Fig 1; Table II). Median plasma aminotransferases and glutamyl transferase activities were greater in patients receiving $\mathrm{PN}$, while median bilirubin levels were in the normal range in both groups (Table II). Of the 30 control liver biopsies, 25 were graded normal, 4 samples displayed mild (grade I) steatosis, and 1 specimen displayed mild (Metavir stage I) fibrosis.

Liver alpha-SMA expression. Quantitative immunohistochemical expression of alpha-SMA showed a similar, 2-fold mean increase in patients on $\mathrm{PN}$ and in patients weaned off PN compared with controls without a significant difference between the 2 IF subgroups (Fig 1). The alpha-SMA expression was most prominent in the portal areas with frequent bridging and occasional extension toward the central veins (Fig 2). RNA expression of ACTA2, encoding for alpha-SMA, was significantly greater in patients on $\mathrm{PN}$ compared with patients weaned off PN or controls (Fig 1). ACTA2 gene expression correlated positively with alphaSMA area fraction $(\mathrm{r}=0.552, P=.006)$.

Liver collagen 1 expression. Similar to alphaSMA, immunohistochemical collagen 1 expression showed a comparable, significant increase in patients on $\mathrm{PN}$ and in patients weaned off $\mathrm{PN}$ compared with controls (Fig 1). The expression pattern of collagen 1 followed that of alpha-SMA (Fig 2). RNA expression of COL1A2, encoding for collagen 1, was not significantly different between the 3 groups (Fig 1). Both protein $(10.3 \pm 0.9$ vs $6.2 \pm 0.8, P=.003)$ and gene expression of collagen $1(1.3 \pm 0.2$ vs $0.7 \pm 0.1, P=.007)$ were greater in patients with histologic liver fibrosis compared with patients without fibrosis. COL1A2 gene expression correlated positively with collagen 1 area fraction $(\mathrm{r}=0.547, P=.007)$ and Metavir fibrosis stage $(\mathrm{r}=0.590, P=.002)$.

Proinflammatory cytokines. Liver RNA expression of the proinflammatory cytokine genes, IL1A and $I L 1 B$, encoding for IL- $1 \alpha$ and IL- $1 \beta$, showed comparable 2- to 5-fold mean increases from control values in patients on $\mathrm{PN}$ and in patients weaned off PN (Fig 3). Expression of ITGB6, encoding for integrin- $\beta 6$, an extracellular matrix adhesion molecule, was similarly 4 - to 5-fold greater in patients on $\mathrm{PN}$ and in patients weaned off PN than it was in controls (Fig 3). Gene expression of tumor necrosis factor $(T N F)$ was not significantly different between patients receiving PN $(1.6 \pm 0.3)$, patients weaned off PN $(1.1 \pm 0.1)$, and controls $(1.2 \pm 0.3$, Kruskal-Wallis $P=.442)$. 
Table II. Liver histology and biochemistry

\begin{tabular}{|c|c|c|c|c|}
\hline Variable & Scale & Patients on PN currently & Patients weaned off $P N$ & $\mathrm{P}$ value \\
\hline Patients, $n$ & & 7 & 18 & \\
\hline \multicolumn{5}{|l|}{ Liver histology } \\
\hline Abnormal liver histology, $n$ & & 6 & 14 & .564 \\
\hline Liver fibrosis, $n$ & & 5 & 12 & $>.99$ \\
\hline Metavir stage* & $0-4$ & $2.0(1.0-2.5)$ & $2.0(1.3-2.0)$ & .646 \\
\hline Portal fibrosis* & $0-4$ & $2.0(1.0-2.5)$ & $2.0(1.25-2.0)$ & .646 \\
\hline Lobular fibrosis* & $0-2$ & $1.0(0.5-1.0)$ & $1.0(0.0-1.0)$ & .419 \\
\hline Steatosis, $n$ & & 5 & 8 & .378 \\
\hline Grade* & $0-3$ & $2.0(1.0-2.5)$ & $1.0(1.0-2.8)$ & .193 \\
\hline Portal inflammation, $n$ & & 1 & 1 & .490 \\
\hline Grade* & $0-3$ & 2.0 & 2.0 & .479 \\
\hline Cholestasis, $n$ & & 1 & 0 & .280 \\
\hline Grade* & $0-3$ & 2.0 & 0 & 109 \\
\hline \multicolumn{5}{|l|}{ Liver biochemistry } \\
\hline Plasma ALT, $n \dagger$ & & 3 & 2 & .113 \\
\hline $\mathrm{U} / \mathrm{L}$ & & $38(32-59)$ & $29(20-35)$ & .039 \\
\hline Plasma AST, $n^{\dagger}$ & & 1 & 2 & $>.99$ \\
\hline $\mathrm{U} / \mathrm{L}$ & & $43(35-49)$ & $35(29-42)$ & .021 \\
\hline Plasma GT, $n_{\dagger}^{\dagger}$ & & 2 & 0 & .070 \\
\hline $\mathrm{U} / \mathrm{L}$ & & $29(21-71)$ & $14(10-21)$ & .003 \\
\hline Plasma bilirubin, $n \dagger$ & & 0 & 1 & $>.99$ \\
\hline$\mu \mathrm{mol} / \mathrm{L}$ & & $3(2-6)$ & $6(5-10)$ & $\begin{array}{l}.028 \\
>99\end{array}$ \\
\hline $\begin{array}{l}\text { Plasma conjugated bilirubin, } n \dagger \\
\mu \mathrm{mol} / \mathrm{L}\end{array}$ & & $\begin{array}{l}1 \\
1(1-2)\end{array}$ & $\begin{array}{c}2 \\
3(1-3)\end{array}$ & $\begin{array}{l}>.99 \\
.095\end{array}$ \\
\hline APRI & & $0.3(0.2-0.4)$ & $0.3(0.2-0.3)$ & .679 \\
\hline
\end{tabular}

*Data for patients with abnormal histology.

$\nmid$ Number of patients off normal range; $P$ value from Mann-Whitney $U$ test between patients on PN and patients weaned off PN.

Data are median (interquartile range) or frequencies.

$P N$, Parenteral nutrition; ALT, alanine aminotransferase; AST, aspartate aminotransferase; GT, glutamyl transferase; APRI, AST-to-platelet ratio index.

Serum IL-1 $\beta$ level was not significantly different between the 3 groups.

Expression of growth factors. RNA expression of EGF, encoding for epidermal growth factor (EGF), was similarly 7 - to 10 -fold greater in patients on PN and in patients weaned off PN than it was in controls (Fig 3). The gene expression of TGFB2, encoding for transforming growth factor (TGF) $\beta 2$, was not significantly different across groups (Kruskal-Wallis $P=.085$, Fig 3 ).

MMPs and their inhibitors. Liver $M M P 9$ gene expression and immunohistochemical expression of TIMP1 were significantly greater in patients on PN compared with controls (Figs 3 and 4). Patients weaned off PN showed increased MMP9 gene expression of similar magnitude in relation to controls (Fig 3), while protein expression of TIMP1 was significantly less in relation to patients on PN and not different from controls (Fig 4). In patients on PN, serum levels of TIMP1 and MMP-7 were significantly greater than in controls (Fig 4). No respective increases were observed in patients weaned off PN, while serum levels of MMP7 were significantly less than in patients on PN. In patients on PN, median levels of serum MMP9 were decreased compared with controls (197 [123255] vs 351 [255-547] ng/mL, $P=.007)$. Serum MMP8 levels were not significantly different between groups.

\section{DISCUSSION}

In this study, we have shown that pediatric-onset IF, both in patients currently on PN and in those weaned off $\mathrm{PN}$, is characterized by abnormal hepatic accumulation of smooth muscle and collagen. Deposition of extracellular matrix in the liver was accompanied by marked transcriptional upregulation of proinflammatory cytokines $I L-1 \alpha$ and $I L-1 \beta$, profibrotic growth factor $E G F$, adhesion molecule integrin- $\beta 6$, and fibrolytic MMP9. The extent of these changes was comparable between patients who were currently receiving PN and those who had been weaned from PN several years earlier. These findings suggest that the liver remains under a persisting proinflammatory and fibrogenic stimulus in association with 
A

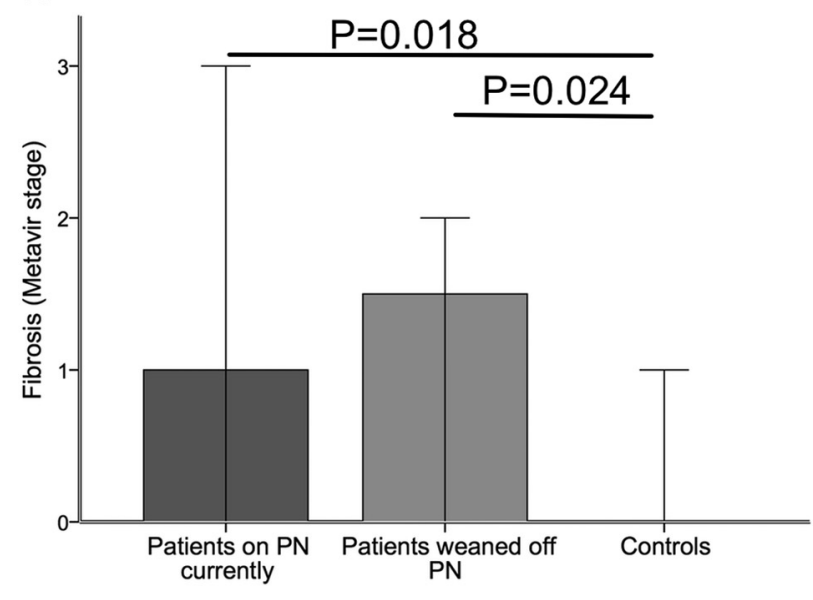

C

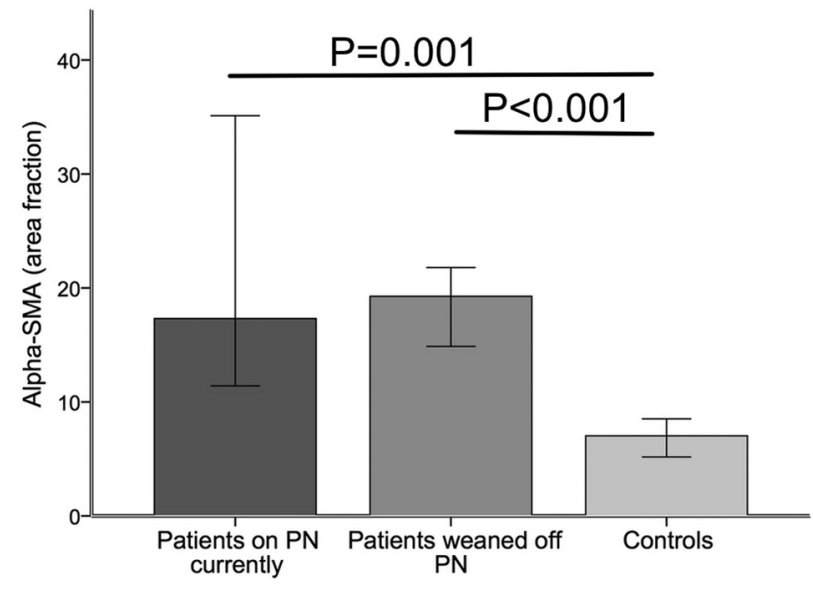

E

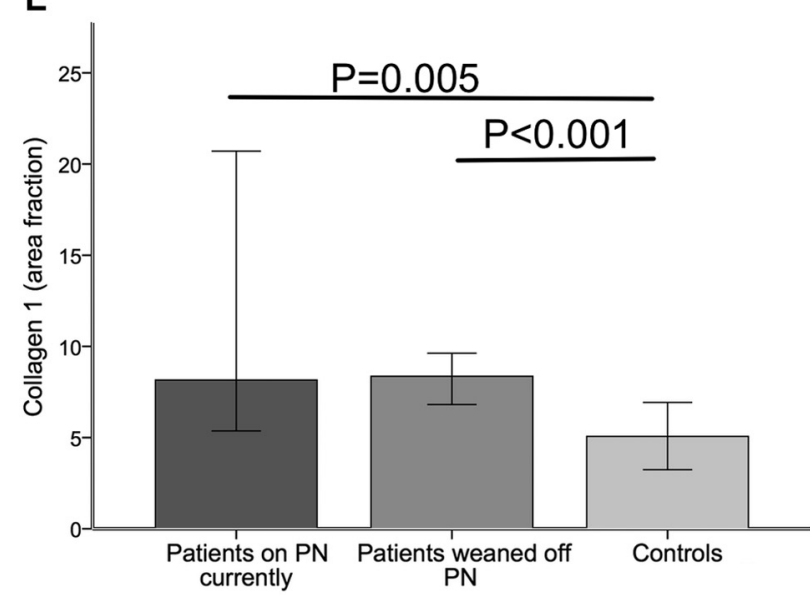

B

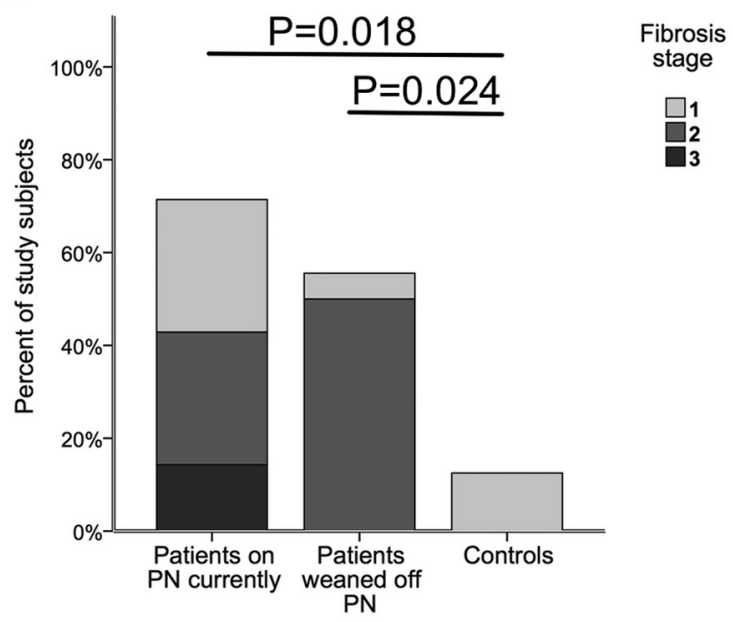

D

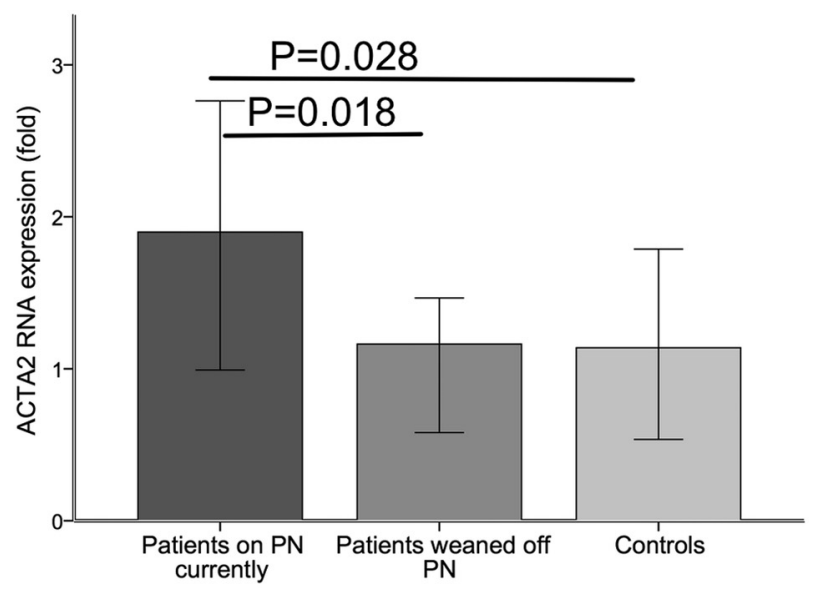

$\mathbf{F}$

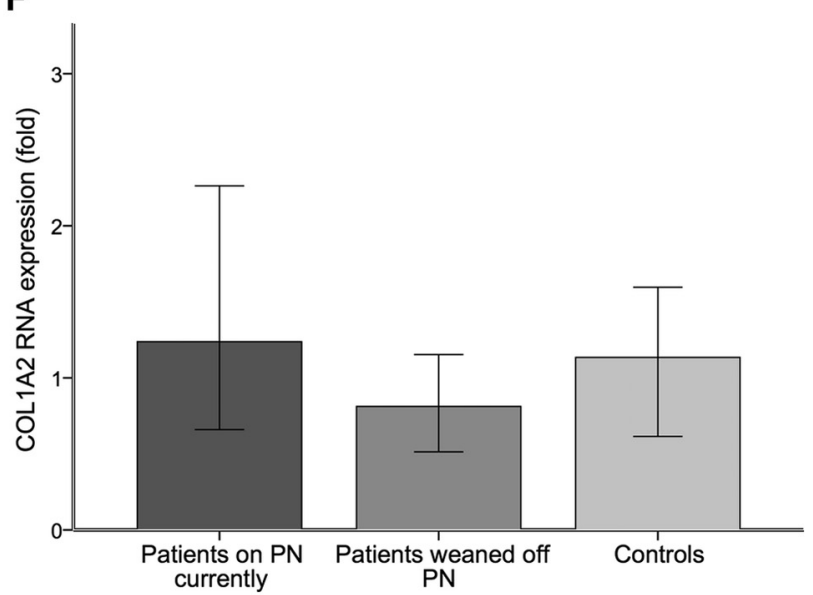

Fig 1. Metavir fibrosis stage $(A)$, distribution of fibrosis stages $(B)$, immunostaining of alpha-SMA $(C)$, liver gene expression of ACTA2 encoding alpha-SMA $(D)$, immunostaining of collagen $1(E)$, and liver gene expression of COLA12 encoding collagen $1(F)$ in patients currently receiving $\mathrm{PN}$, patients weaned off $\mathrm{PN}$, and controls. Immunostaining is expressed as percentage proportion of stained area and gene expression as fold-change relative to controls. KruskalWallis test for between groups comparisons followed by Mann-Whitney $U$ test for pairwise comparisons. Median values and $95 \%$ confidence intervals or frequencies are shown. 

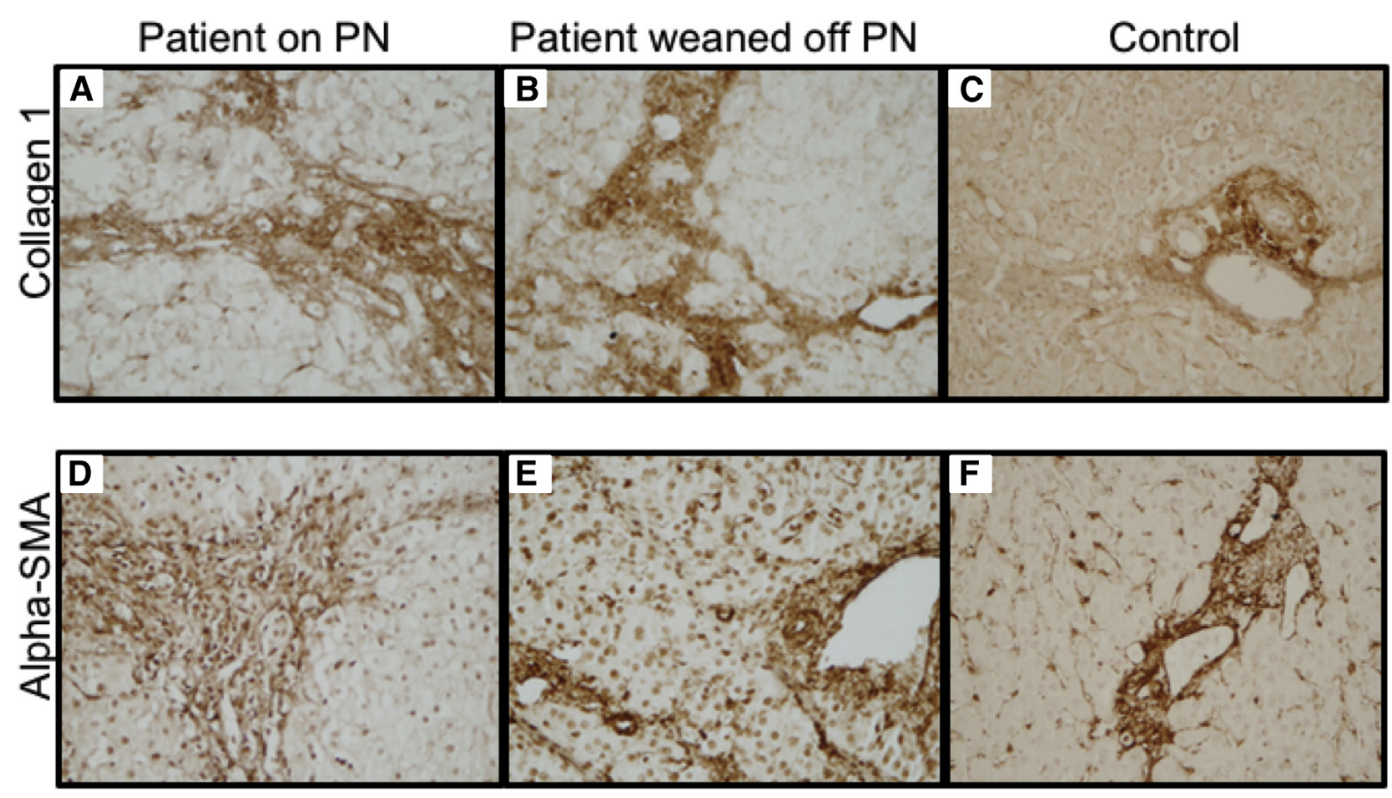

Fig 2. Representative examples of liver collagen $1(A-C)$ and alpha-SMA $(D-F)$ immunostaining in patients currently receiving PN, patients weaned off $\mathrm{PN}$, and control patients $(20 \times$ magnification). Collagen 1 and alpha-SMA expression were increased similarly in both patient groups being most prominent in the portal areas with frequent bridging and occasional extension toward central veins.

activation of myofibroblastic cells and accumulation of extracellular matrix also after weaning off $\mathrm{PN}$, presumably because of ongoing disturbed intestinal homeostasis in patients with compensated short bowel syndrome and severe motility disorders that led to IF.

Administration of PN and impaired intestinal barrier function are required for development of cholestatic liver injury in mice. ${ }^{5}$ In this novel mouse model, pharmacologically induced intestinal barrier dysfunction in intact intestine leads to lipopolysaccharide translocation to the portal vein, which in combination with PN containing the plant sterol (stigmasterol), activates Kupffer cell expression of the proinflammatory cytokines IL-1, IL-6, and TNF $\alpha$ and the profibrotic TGF $\beta$ through toll-like receptor 4 signaling. ${ }^{4,5}$ These mechanisms may be translatable to IFALD observed in humans.

Previous clinical studies support the role of plant sterols in PN and intestinal barrier dysfunction in the pathogenesis of IFALD after prolonged duration of PN required for survival. The lipids in PN-containing plant sterols and their increased serum concentrations have been linked repeatedly with biochemical and histologic liver injury in children and adults. ${ }^{18-22}$ Children with IF display an overabundance of lipopolysaccharide-producing Proteobacteria in their intestinal microbiota in association with histologic liver fibrosis and steatosis, intestinal inflammation, and increased serum levels of IL- 6 and TNF $\alpha,{ }^{6,11,23}$ while other clinical studies indicate that short bowel syndrome promotes intestinal permeability and continuous systematic exposure to lipopolysaccharides. ${ }^{24-26}$

In the present study, the combination of intestinal compromise (IF) and PN was associated with markedly increased hepatic gene expression of $I L-1 \alpha, I L-1 \beta, E G F$, integrin- $\beta 6$, and MMP-9, although liver $T G F$ and $T N F$ gene expression remained statistically insignificant. These cytokines, growth factors, adhesion molecules, and metalloproteinases are key effectors in inflammation pathways driving liver fibrogenesis and extracellular matrix remodeling, ${ }^{13}$ as reflected by increased smooth muscle gene, protein expression, and collagen accumulation in the present study. Notably, MMP9 is expressed by IL-1-activated Kupffer cells, inflammatory macrophages, and hepatic stellate cells and is a principal mediator of stellate cell activation. ${ }^{27}$ Hepatic expression of $M M P 9$ and $E G F$ are both related to progression of liver fibrosis in humans. ${ }^{27,28}$

In pediatric-onset IF, histologic liver fibrosis and steatosis are interrelated and persist for more than a decade after cessation of PN, possibly representing a permanent change. ${ }^{8,29}$ Whether the persistent histologic injury represents a progressive disease process has become relevant, because an increasing number of children with neonatal IF 
A

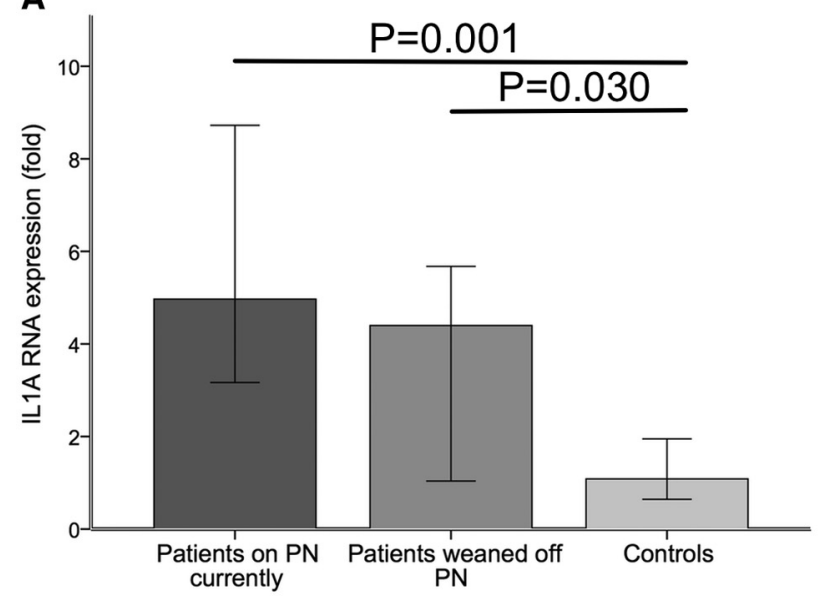

C

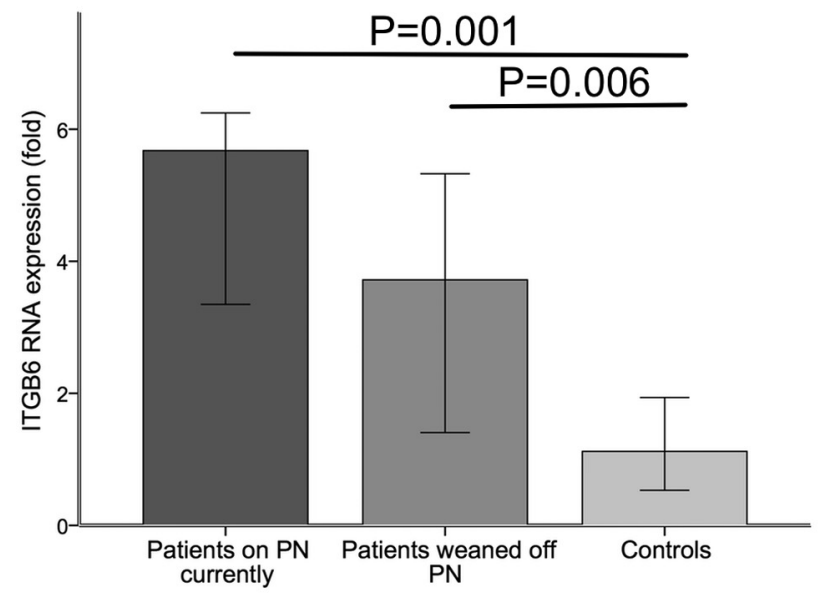

E

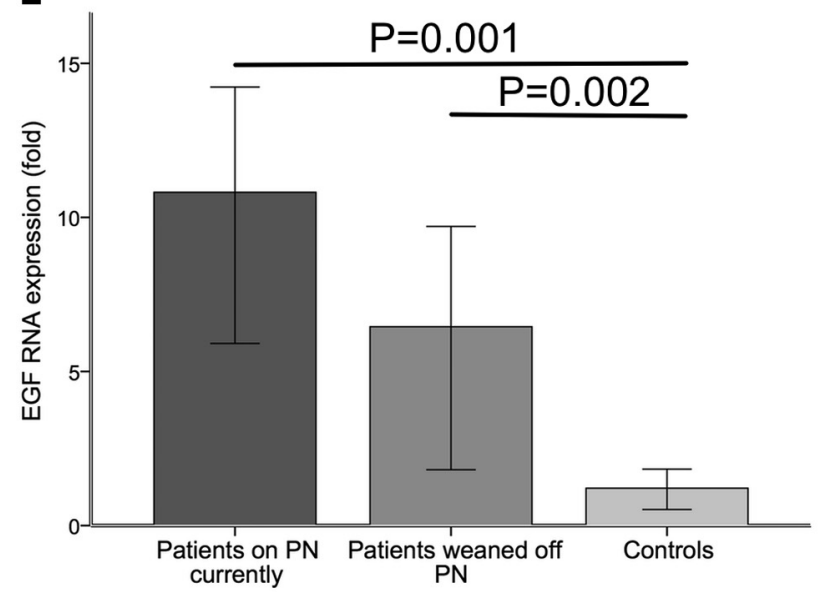

B

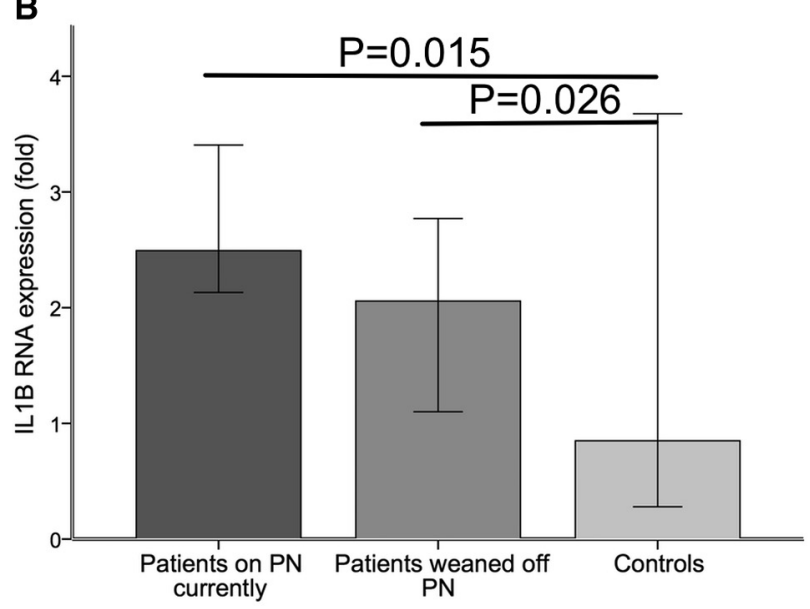

D

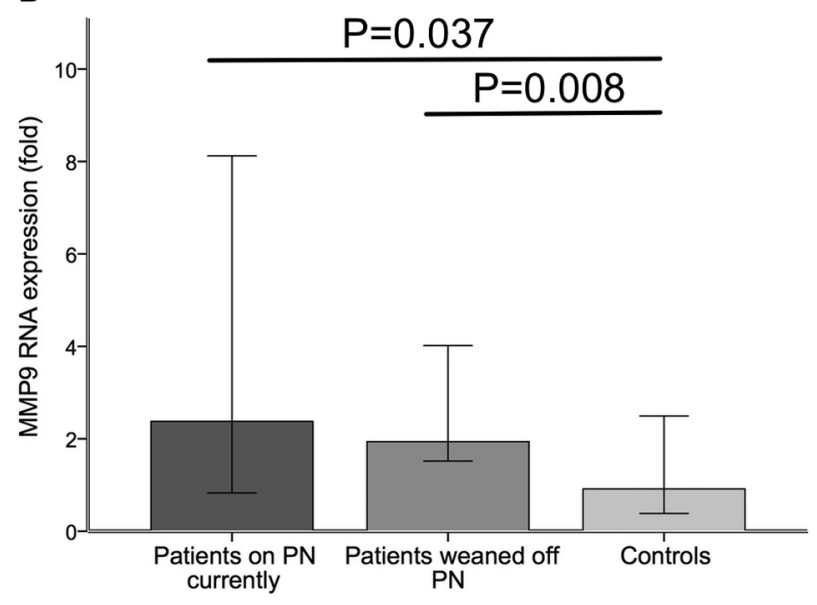

$\mathbf{F}$

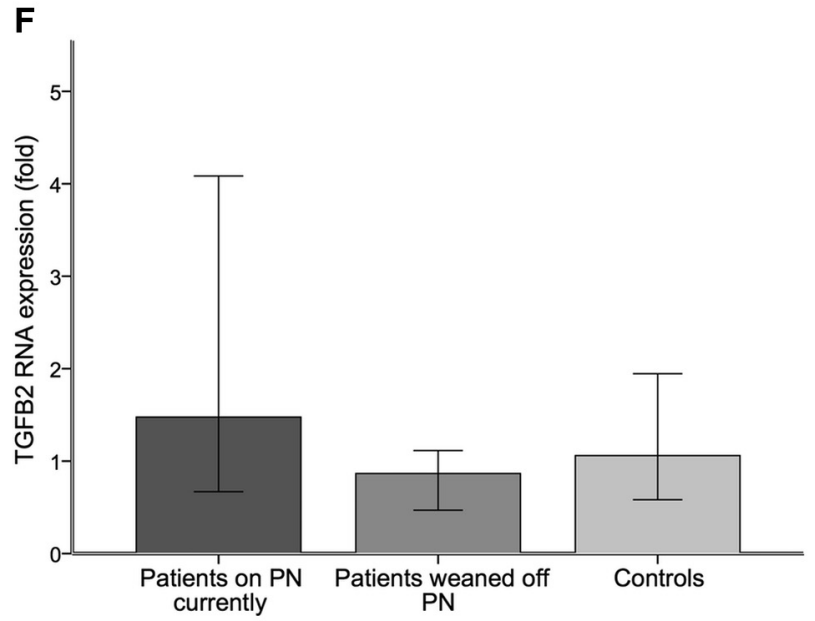

Fig 3. Relative liver gene expression of $\operatorname{IL1A}(A), \operatorname{IL1B}(B), \operatorname{ITGB6}(C), M M P 9(D), E G F(E)$, and TGFB2 (F) in patients currently receiving PN, patients weaned off PN, and control patients. Immunostaining is expressed as percentage proportion of stained area and gene expression as fold-change relative to controls. Kruskal-Wallis test for between-group comparisons followed by Mann-Whitney $U$ test for pairwise comparisons. Median and $95 \%$ confidence intervals are shown. 
A

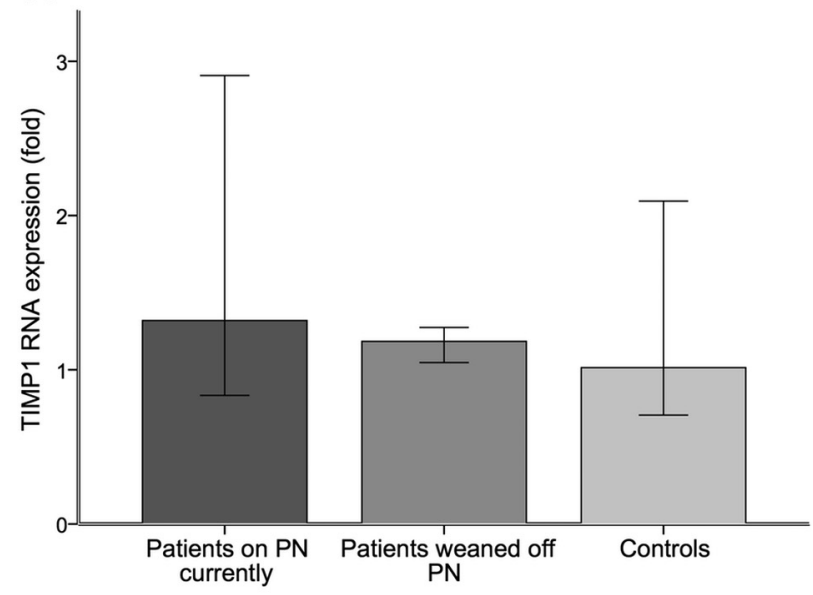

C

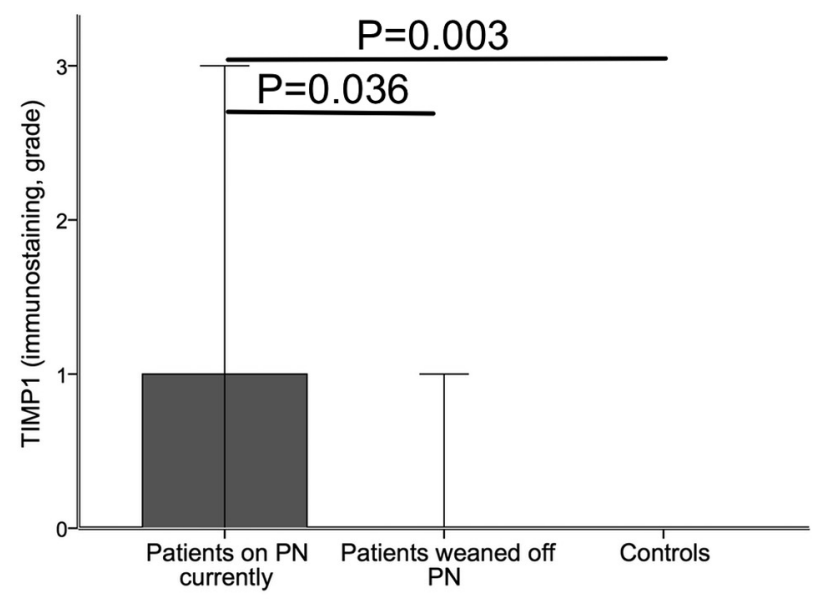

E

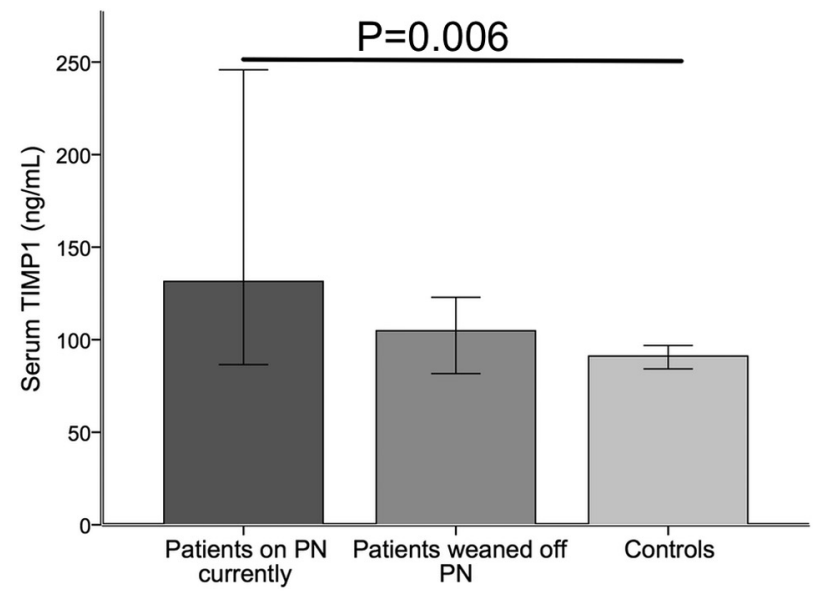

B

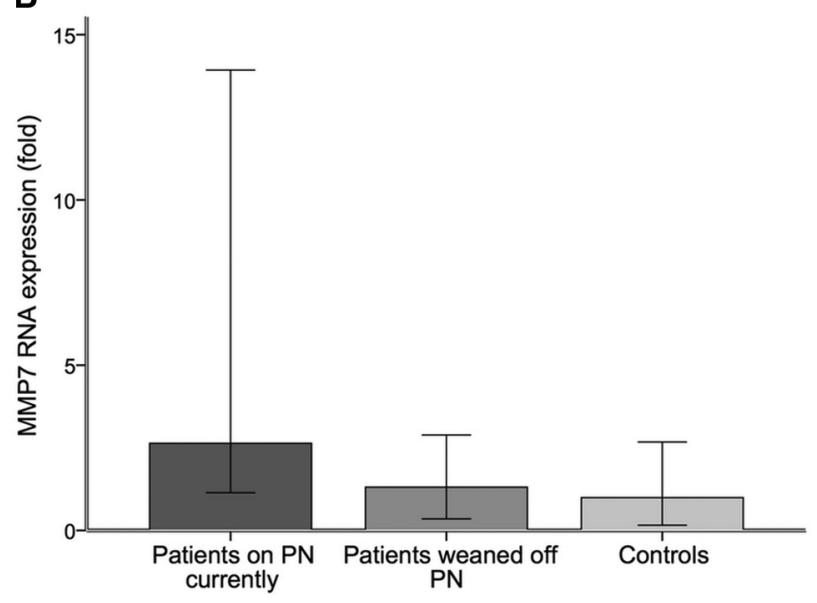

D

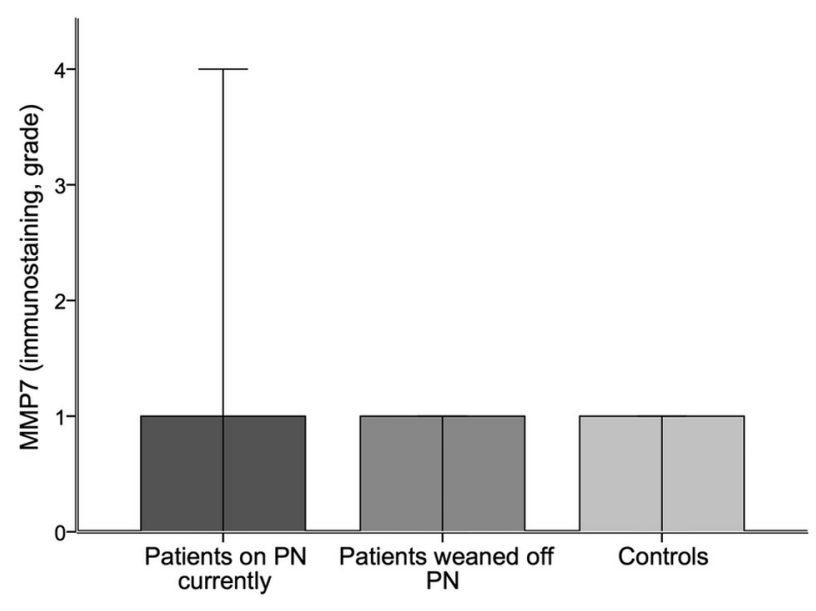

$\mathbf{F}$

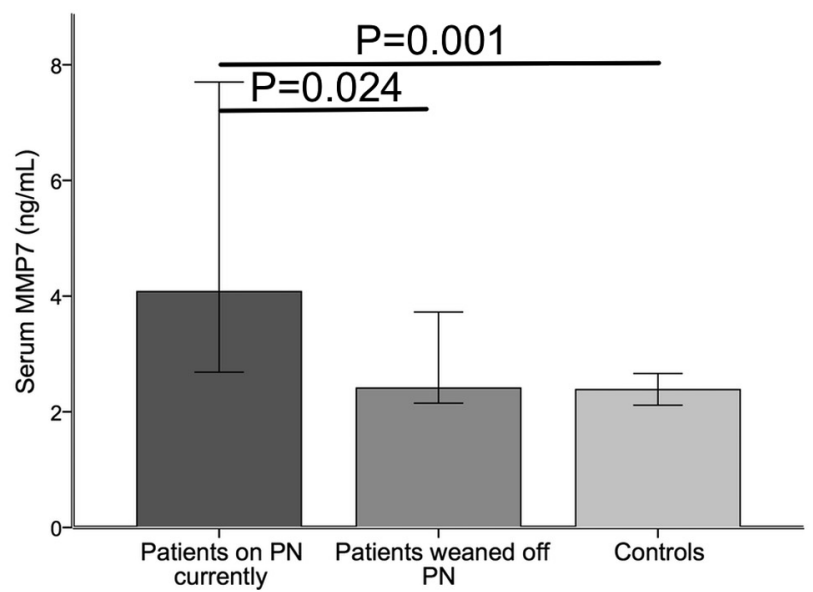

Fig 4. Relative liver gene expression, immunohistochemistry, and serum levels of TIMP1 $(A, C, E)$ and MMP7 $(B, D, F)$ in patients currently receiving PN, patients weaned off PN, and controls. Immunostaining of TIMP1 in perisinusoidal hepatocytes and MMP7 in bile ducts were graded visually from 0-3. Gene expression is fold-change relative to controls. Kruskal-Wallis test for between-group comparisons followed by Mann-Whitney $U$ test for pairwise comparisons. Median and $95 \%$ confidence intervals are shown. 
are weaned off PN successfully with a long life expectancy. ${ }^{9,10,30,31}$

In the present study, the comparable degree of hepatic smooth muscle expression, collagen accumulation, and transcriptional upregulation of $I L-1$, $E G F$, integrin- $\beta 6$, and $M M P 9$ between patients who were receiving PN currently and those who had been weaned from PN for a median of 6 years before, suggests first that compromised intestinal function may contribute to the pathogenesis of IFALD possibly by providing a persistent proinflammatory and profibrotic stimulus to the liver by multiple potential mechanisms, ${ }^{32}$ and secondly, that the liver may remain under an ongoing, injurious stimulus after weaning off $\mathrm{PN}$.

These presumptions are supported by previous experimental and clinical observations. Extensive small intestinal resection alone in pigs without simultaneous PN causes histologic liver fibrosis and steatosis together with increased hepatic expression of proinflammatory cytokines in association with an altered intestinal microbiota, bile acid metabolism, and farnesoid receptor X $(F R X)$ signaling. ${ }^{12}$ In children with IF, loss of the distal small intestine (ileum) irrespective of PN delivery correlates closely with histologic liver fibrosis and decreased levels of circulating fibroblast growth factor (FGF) 19.

An impaired FXR-FGF 19 axis has the potential to promote liver injury via a multitude of mechanisms by interfering with the control of bile acid homeostasis, biliary secretion, lipid metabolism, and inflammation in the liver and by failing to prevent bacterial overgrowth and intestinal barrier dysfunction in the gut. ${ }^{33-35}$ Striking alterations in the intestinal microbiota, such as an overabundance of lactobacilli, especially after weaning off PN, may interfere further with the regulation of bile acids and subsequent hepatic and intestinal function induced by chemical modification of bile acids, altering their ability to activate target receptors. ${ }^{11,36}$

We observed some notable differences during PN administration and after weaning off PN. Upregulation of liver $\alpha-S M A$ gene expression and expression of TIMP1 and MMP7 in liver and serum occurred only in patients who were receiving PN currently. In general, these findings point to a greater activity of liver fibrogenesis when IFassociated intestinal compromise was combined with $\mathrm{PN}$, supporting their synergistic role in the pathogenesis of IFALD $^{13,27}$ in accordance with experimental findings. ${ }^{5}$

Our study had some limitations. In addition to the limited number of patients, the cross-sectional design provides associations rather than proven causal relationships between different study variables. Other challenges in our study design include the wide age range of the patients and the evolution of treatment strategies of IF patients over time. Composition of parenteral lipids has changed from soy-based to olive oil- and fish oilbased lipids; the amount of PN fat is limited; and early initiation of enteral nutrition and cyclic PN infusions are introduced actively. Although the changes in clinical practice may have modulated our findings and may hamper their applicability for newly treated children with IF, our study provides reliable information regarding the current, long-term features of liver remodeling in IFALD.

It is also unlikely that the described treatment changes will affect the liver after weaning off PN. Due to limited availability of healthy liver biopsy material for obvious reasons, our control material was obtained from 3 different subgroups; all subgroups, however, matched for age with patients. It may also be argued that donor livers or patients operated on for complicated cholelithiasis do not represent normal controls, but only very few of their liver biopsies ( 5 of 30 biopsies) revealed minor histologic changes.

In conclusion, this study shows that pediatriconset IF is characterized by abnormally increased hepatic smooth muscle expression and collagen accumulation in IF patients receiving PN currently and after weaning off PN. These changes were accompanied with increased gene expression of selected proinflammatory and fibrogenic cytokines both during PN delivery and after weaning off PN. Persistent proinflammatory and fibrogenic transcriptional activation after weaning off $\mathrm{PN}$ provides indirect clinical mechanistic evidence that factors other than PN, possibly intestinal compromise in IF, may contribute to pathogenesis of IFALD. Based on these results, clinical mechanistic studies on the contribution of liver-gut axis to pathogenesis of IFALD are needed. In addition, long-term follow-up studies on liver function in pediatric IF patients after weaning off $\mathrm{PN}$ are warranted.

\section{REFERENCES}

1. Lauriti G, Zani A, Aufieri R, Cananzi M, Chiesa PL, Eaton S, et al. Incidence, prevention, and treatment of parenteral nutrition-associated cholestasis and intestinal failureassociated liver disease in infants and children: A systematic review. JPEN J Parenter Enteral Nutr 2014;38:70-85.

2. Lacaille F, Gupte G, Colomb V, D'Antiga L, Hartman C, Hojsak I, et al. Intestinal failure - associated liver disease. A position paper by the ESPGHAN Working Group of Intestinal Failure and Intestinal Transplantation. J Pediatr Gastroenterol Nutr 2015;60:272-83. 
3. Wales PW, Allen N, Worthington P, George D, Compher C, American Society for Parenteral and Enteral Nutrition, et al. A.S.P.E.N. clinical guidelines: Support of pediatric patients with intestinal failure at risk of parenteral nutritionassociated liver disease. JPEN J Parenter Enteral Nutr 2014;38:538-57.

4. El Kasmi KC, Anderson AL, Devereaux MW, Vue PM, Zhang W, Setchell KD, et al. Phytosterols promote liver injury and Kupffer cell activation in parenteral nutritionassociated liver disease. Sci Transl Med 2013;5:206ra137.

5. El Kasmi KC, Anderson AL, Devereaux MW, Fillon SA, Harris JK, Lovell MA, et al. Toll-like receptor 4-dependent Kupffer cell activation and liver injury in a novel mouse model of parenteral nutrition and intestinal injury. Hepatology 2012;55:1518-28.

6. Mutanen A, Lohi J, Heikkilä P, Jalanko H, Pakarinen MP. Loss of ileum decreases serum fibroblast growth factor 19 in relation to liver inflammation and fibrosis in pediatric onset intestinal failure. J Hepatol 2015;62:1391-7.

7. Lee W, Sokol R. Intestinal microbiota, lipids, and the pathogenesis of intestinal failure-associated liver disease. J Pediatr 2015;167:519-26.

8. Mutanen A, Lohi J, Heikkilä P, Koivusalo AI, Rintala RJ, Pakarinen MP. Persistent abnormal liver fibrosis after weaning off parenteral nutrition in pediatric intestinal failure. Hepatology 2013;58:729-38.

9. Merras-Salmio L, Pakarinen M. Refined multidisciplinary protocol-based approach to short bowel syndrome improves outcomes. J Pediatr Gastroenterol Nutr 2015;61:24-9.

10. Avitzur Y, Wang J, de Silva NT, Burghardt KM, DeAngelis M, Grant D, et al. Impact of intestinal rehabilitation program and its innovative therapies on the outcome of intestinal transplant candidates. J Pediatr Gastroenterol Nutr 2015; 61:18-23.

11. Korpela K, Mutanen A, Salonen A, Savilahti E, de Vos VM, Pakarinen MP. Intestinal microbiota signatures associated with histological liver steatosis in pediatric-onset intestinal failure. JPEN J Parenter Enteral Nutr 2015 May 1. [Epub ahead of print].

12. Pereira-Fantini P, Lapthorne S, Joyce S, Dellios NL, Wilson G, Fouhy F, et al. Altered FXR signalling is associated with bile acid dysmetabolism in short bowel syndromeassociated liver disease. J Hepatol 2014;61:1115-25.

13. Hernandez-Gea V, Friedman S. Pathogenesis of liver fibrosis. Annu Rev Pathol 2011;6:425-56.

14. Struijs M, Diamond I, de Silva N, Wales PW. Establishing norms for intestinal length in children. J Pediatr Surg 2009;44:933-8.

15. Wai C, Greenson JK, Fontana RJ, Kalbfleisch JD, Marrero JA, Conjeevaram HS, et al. A simple noninvasive index can predict both significant fibrosis and cirrhosis in patients with chronic hepatitis C. Hepatology 2003;38:518-26.

16. Rautelin H, Tervahartiala T, Lauhio A, Sorsa T, Kolho KL. Assessment of systemic matrix metalloproteinase and their regulator response in children with Helicobacter pylori gastritis. Scand J Clin Lab Invest 2010;70:492-7.

17. Tuomainen AM, Nyyssönen K, Laukkanen JA, Tervahartiala T, Tuomainen TP, Salonen JT, et al. Serum matrix metalloproteinase- 8 concentrations are associated with cardiovascular outcome in men. Arterioscler Thromb Vasc Biol 2007;27:2722-8.

18. Clayton PT, Bowron A, Mills KA, Massoud A, Casteels M, Milla PJ. Phytosterolemia in children with parenteral nutrition-associated cholestatic liver disease. Gastroenterology 1993;105:1806-13.
19. Kurvinen A, Nissinen MJ, Andersson S, Korhonen P, Ruuska T, Taimisto M, et al. Parenteral plant sterols and intestinal failure-associated liver disease in neonates. J Pediatr Gastroenterol Nutr 2012;54:803-11.

20. Mutanen A, Nissinen MJ, Lohi J, Heikkilä P, Gylling H, Pakarinen MP. Serum plant sterols, cholestanol, and cholesterol precursors associate with histological liver injury in pediatric onset intestinal failure. Am J Clin Nutr 2014;100:1085-94.

21. Llop JM, Virgili N, Moreno-Villares JM, García-Peris P, Serrano T, Forga M, et al. Phytosterolemia in parenteral nutrition patients: Implications for liver disease development. Nutrition 2008;24:1145-52.

22. Zaloga G. Phytosterols, lipid administration, and liver disease during parenteral nutrition. JPEN J Parenter Enteral Nutr 2015;39:39S-60S.

23. Kaufman SS, Loseke CA, Lupo JV, Young RJ, Murray ND, Pinch LW, et al. Influence of bacterial overgrowth and intestinal inflammation on duration of parenteral nutrition in children with short bowel syndrome. J Pediatr 1997;131:356-61.

24. D’Antiga L, Dhawan A, Davenport M, Mieli-Vergani G, Bjarnason I. Intestinal absorption and permeability in paediatric short-bowel syndrome: A pilot study. J Pediatr Gastroenterol Nutr 1999;29:588-93.

25. Ziegler TR, Luo M, Estívariz CF, Moore DA 3rd, Sitaraman SV, Hao L, et al. Detectable serum flagellin and lipopolysaccharide and upregulated anti-flagellin and lipopolysaccharide immunoglobulins in human short bowel syndrome. Am J Physiol Regul Integr Comp Physiol 2008; 294:R402-10.

26. Ralls MW, Demehri FR, Feng Y, Woods Ignatoski KM, Teitelbaum DH. Enteral nutrient deprivation in patients leads to a loss of intestinal epithelial barrier function. Surgery 2015;157:732-42.

27. Hemmann S, Graf J, Roderfeld M, Roeb E. Expression of MMPs and TIMPs in liver fibrosis - A systematic review with special emphasis on anti-fibrotic strategies. J Hepatol 2007;46:955-75.

28. Fuchs BC, Hoshida Y, Fujii T, Wei L, Yamada S, Lauwers GY, et al. Epidermal growth factor receptor inhibition attenuates liver fibrosis and development of hepatocellular carcinoma. Hepatology 2014;59:1577-90.

29. Mutanen A, Heikkilä P, Lohi J, Raivio T, Jalanko $H$, Pakarinen MP. Serum FGF21 increases with hepatic fat accumulation in pediatric onset intestinal failure. J Hepatol 2014;60:183-90.

30. Modi BP, Langer M, Ching YA, Valim C, Waterford SD, Iglesias $\mathrm{J}$, et al. Improved survival in a multidisciplinary short bowel syndrome program. J Pediatr Surg 2008;43:20-4.

31. Khan FA, Squires RH, Litman HJ, Balint J, Carter BA, Fisher JG, et al. Predictors of enteral autonomy in children with intestinal failure: A multicenter cohort study. J Pediatr 2015;167:29-34.

32. Chassaing B, Etienne-Mesmin L, Gewirtz A. Microbiota-liver axis in hepatic disease. Hepatology 2014;59:328-39.

33. Matsubara T, Li F, Gonzalez FJ. FXR signaling in the enterohepatic system. Mol Cell Endocrinol 2013;368:17-29.

34. van Erpecum K, Schaap F. Intestinal failure to produce FGF19: A culprit in intestinal failure-associated liver disease? J Hepatol 2015;62:1231-3.

35. Jahn D, Rau M, Hermanns HM, Geier A. Mechanisms of enterohepatic fibroblast growth factor 15/19 signaling in health and disease. Cytokine Growth Factor Rev 2015;26: 625-35.

36. de Aguiar Vallim T, Tarling E, Edwards P. Pleiotropic roles of bile acids in metabolism. Cell Metab 2013;17:657-69. 